\title{
Impacts de la Recharge sur la Piezometrie et la Mineralisation de la Nappe Phreatique du Kori de Dantiandou (Sud-Ouest Niger)
}

\author{
Salifou Bachir, \\ Département de Géologie, FAST, Université Abdou Moumouni de Niamey, \\ Niger, Institut de Recherche pour le Développement, Niamey, Niger \\ Boureima Ousmane, \\ Département de Géologie, FAST, \\ Université Abdou Moumouni de Niamey, Niger
}

Doi:10.19044/esj.2019.v15n24p262 URL:http://dx.doi.org/10.19044/esj.2019.v15n24p262

Résumé

La nappe phréatique du Kori de Dantiandou est située entre $13^{\circ} 10^{\prime}$ et $13^{\circ} 30^{\prime}$ de latitude Nord et entre $2^{\circ} 40^{\prime}$ et $3^{\circ}$ de longitude Est. Ses réserves en eau sont annuellement renouvelées comme le confirment les observations de terrain conduites depuis plus de 25 ans (programmes de recherche internationaux Hapex Sahel puis AMMA) qui ont montré qu'en dépit des grandes sècheresses au Sahel (1970 - 1980) la nappe phréatique a connu une remontée continue d'environ $4 \mathrm{~m}$ en 40 ans. Cependant, cette recharge expose la nappe à une évaporation et une salinisation. L'objectif principal de cette étude est de préciser la dynamique de la nappe et ses impacts souvent négatifs sur la salinité des ressources en eau, limitant ainsi certains de leurs usages. L'approche méthodologique basée sur l'analyse des niveaux piézométriques, l'étude des paramètres physicochimiques et l'utilisation des isotopes a permis d'obtenir plusieurs résultats importants pour la disponibilité future d'eau douce dans la région. Une hausse généralisée des réserves souterraines s'observe, se traduisant par l'apparition de nouvelles mares permanentes dans les zones les plus basses. La reprise évaporatoire directe à partir de la nappe augmente considérablement la conductivité électrique (CE supérieures 1000 $\left.\mu \mathrm{S} . \mathrm{cm}^{-1}\right)$, tandis que la minéralisation naturelle des eaux souterraines reste très basse (CE entre 20 et $\left.100 \mu \mathrm{S} . \mathrm{cm}^{-1}\right)$. Des enrichissements très importants des teneurs en isotopes stables $(\mathrm{O}-18$ et $\mathrm{D})$ confirment la forte minéralisation liée à l'évaporation. Enfin, il est observé une salinisation des eaux et des sols qui limite les usages des ressources disponibles, malgré leur abondance.

Mots-clés : Kori de Dantiandou, Recharge, Minéralisation, Isotopes stables, Evaporation 


\title{
Impacts of Recharge on Piezometry and Mineralisation of the Dantiandou Kori Water Table (South-West Niger)
}

\author{
Salifou Bachir, \\ Département de Géologie, FAST, Université Abdou Moumouni de Niamey, \\ Niger, Institut de Recherche pour le Développement, Niamey, Niger \\ Boureima Ousmane, \\ Département de Géologie, FAST, \\ Université Abdou Moumouni de Niamey, Niger
}

\begin{abstract}
The water table of the Dantiandou Valley is located between $13^{\circ} 10^{\prime}$ and $13^{\circ} 30^{\prime}$ north latitude and between $2^{\circ} 40^{\prime}$ and $3^{\circ}$ east longitude. Its water reserves are renewed annually as confirmed by long-term observations conducted over more than 25 years (international experiments Hapex Sahel and AMMA) having shown that the water table has experienced a continuous rise of about $4 \mathrm{~m}$ in 40 years despite the severe droughts in the Sahel (1970 1980). However, this recharge exposes the water table to evaporation and salinization. The main objective of this study is to confirm the dynamics of the water table and its often negative impacts on the salinity of water resources, thus limiting some of their uses. The methodological approach based on analysis of piezometric levels, study of physicochemical parameters and use of isotopes has yielded several important results for future freshwater availability in the region. General increase in groundwater reserves has resulted in the emergence of new permanent ponds in the lowest valley bottoms. Evaporation strongly affects the water table and increases significantly its electrical conductivity (EC up to $1000 \mu \mathrm{S} . \mathrm{cm}^{-1}$ ) while the natural mineralization of groundwater is very low (EC between 20 and 100 $\left.\mu \mathrm{S} . \mathrm{cm}^{-1}\right)$. Very important enrichments of the stable isotope (O-18 and D) contents confirm the increase in salinity due to evaporation. Finally, salinization of water and soil is observed and limits the uses of water resources despite their abundance.
\end{abstract}

Keywords: Kori of Dantiandou, Recharge, Mineralization, Stable isotopes, Evaporation 


\section{Introduction}

Le Niger est un pays désertique sur les $3 / 4$ de sa superficie qui reçoit moins de $150 \mathrm{~mm} . \mathrm{a}^{-1}$. Sur le $1 / 4$ restant, les hauteurs pluviométriques varient de 150 à $800 \mathrm{~mm} \cdot \mathrm{a}^{-1}$. Les eaux de surface sont limitées et rapidement reprises par l'évaporation, en dehors du Fleuve Niger qui est permanent, de la Komadougou Yobé qui est semi-permanente et de quelques mares et lacs permanents. Cependant, le pays renferme d'importantes ressources en eau souterraine contenues dans des aquifères à nappe libre, captive et semicaptive. Les nappes libres, comme celle du Kori de Dantiandou, jouent un rôle important dans l'approvisionnement en eau de la population, l'abreuvement des bétails et l'irrigation.

Dans les régions semi-arides, l'occupation des sols a été souvent citée comme un facteur beaucoup plus important que le climat pour expliquer les changements dans la recharge et la circulation des flux souterrains (Cuthbert et al., 2019 ; DeFries et Eshleman, 2004 ; Leduc et al., 2017 ; Scanlon et al., 2007 ; Vörösmarty et al., 2000). Dans le Sud-Ouest Niger, un exemple très détaillé et documenté de l'impact du déboisement sur la recharge de la nappe du Continental Terminal du bassin des Iullemmeden est fourni par Leduc et al., (2001) et Favreau et al., (2009), qui ont mis en évidence une hausse de 4 $\mathrm{m}$ du niveau moyen de la nappe, malgré les déficits pluviométriques fréquents observés depuis les années 70. Plusieurs études associent cette hausse au déboisement des savanes naturelles qui a provoqué un encroûtement des sols et un afflux d'eau de ruissellement supplémentaire vers les mares et les basfonds endoréiques, considérés comme les principales zones de recharge de l'aquifère (Desconnets et al., 1997 ; Leblanc et al., 2008 ; Martin-Rosales \& Leduc, 2003 ; Massuel et al., 2006).

L’objectif principal de cette étude, est de préciser la dynamique à long terme de la nappe phréatique du Kori de Dantiandou en zone sahélienne. La méthode utilisée repose sur l'étude de la remontée piézométrique, des variations des paramètres isotopiques et chimiques ainsi que des impacts de la variabilité de ces paramètres sur l'aptitude des eaux aux différents usages.

\section{Cadre de l'étude}

La zone d'étude, située à l'aval du kori de Dantiandou, dans l'extrémité sud du bassin des Iullemmeden qui occupe la partie sud-ouest du Niger, est caractérisée par l'apparition récente de plusieurs mares permanentes dans les zones basses (Fig. 1). 


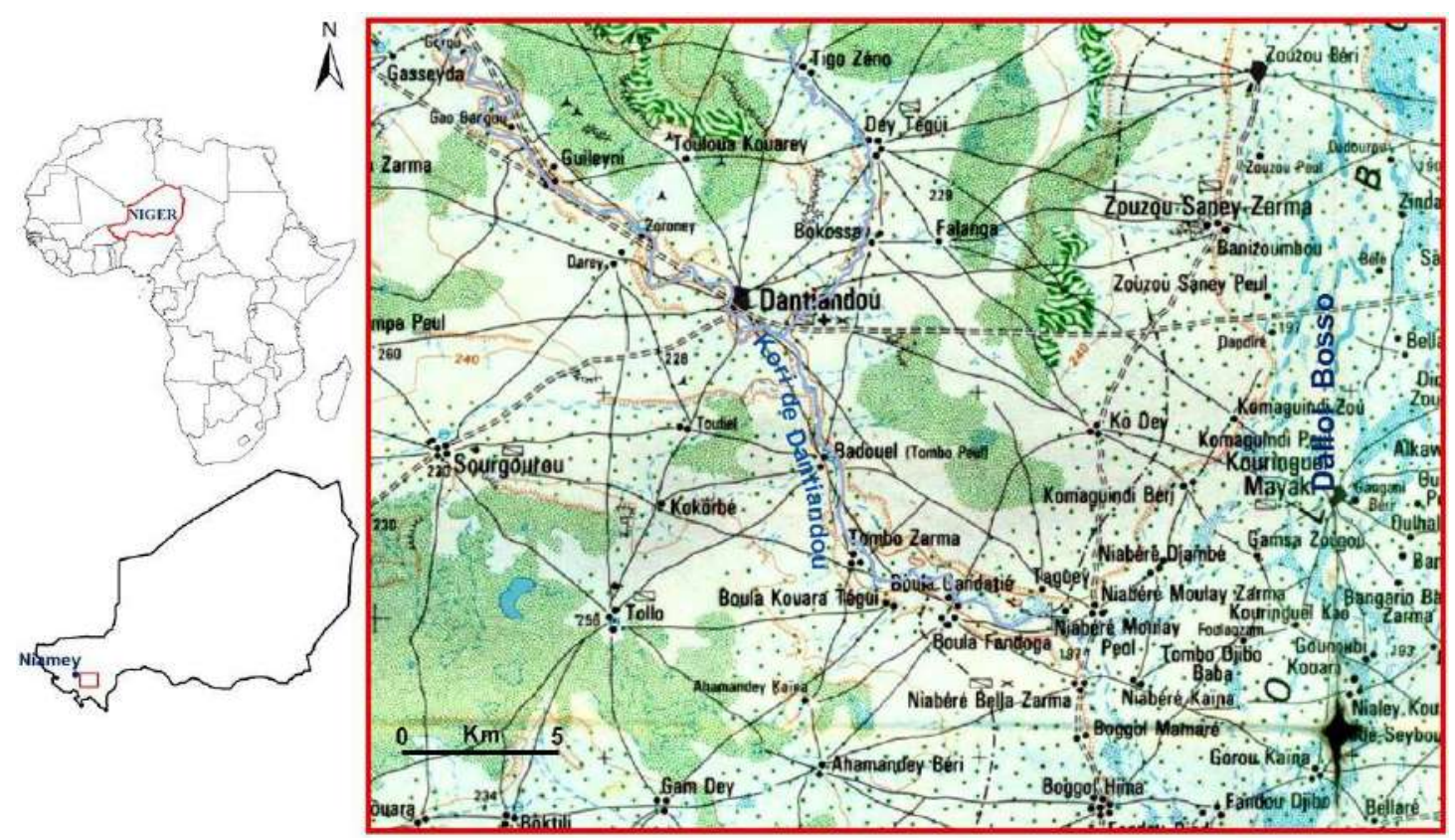

Figure 1. Contexte géographique de la zone d'étude

La population de la zone est essentiellement rurale, et elle est rassemblée en de nombreux petits villages de quelques centaines d'habitants. La croissance démographique de cette population, qui était de $1,5 \% \mathrm{a}^{-1}$ en 1950, est passée à 3,9\% $\mathrm{a}^{-1}$ en 2012 (INS, 2013), constituant l'une des plus fortes croissances démographiques au monde. Cette population vit essentiellement de l'agriculture pluviale, dont les principales spéculations sont le mil et le sorgho. Cependant, dans les bas-fonds humides, le riz, l'arachide, le manioc et les patates douces sont cultivés. De même, on observe un développement de l'arboriculture (manguiers, citronniers, palmiers, ... etc.)

\section{Contexte hydro-climatique}

Le climat, de type sahélien, est semi-aride et caractérisé par deux saisons : une longue saison sèche de 7 à 8 mois, débutant en octobre-novembre et se terminant en avril-mai, à laquelle succède une saison humide (hivernage) courte, de 4 à 5 mois. La moyenne interannuelle des précipitations enregistrées à Niamey entre 1905 et 2015 est de l'ordre de $560 \mathrm{~mm}$ avec un écart type de $135 \mathrm{~mm}$ (Niamey Aéroport, Direction de la Météorologie Nationale du Niger). Elle peut être considérée comme représentative de la zone d'étude. Les $90 \%$ de ces précipitations tombent dans les mois de juillet/août, avec des années sèches comme humides. La température moyenne annuelle est de $29^{\circ} \mathrm{C}$. Les mois de novembre à février sont caractérisés par des températures fraiches pouvant descendre jusqu'à $10{ }^{\circ} \mathrm{C}$. Par contre, les mois d'avril et mai ont des températures élevées pouvant dépasser $45^{\circ} \mathrm{C}$ à l'ombre. La hauteur moyenne 
annuelle de l'évapotranspiration potentielle est d'environ $2500 \mathrm{~mm}$, dépassant de près de 4 fois la hauteur moyenne des précipitations. L'humidité relative de l'air, liée à l'avancée du front intertropical, présente des différences saisonnières encore plus marquées. En effet, les valeurs maximales de l'humidité enregistrées pendant la saison des pluies peuvent dépasser $70 \%$, tandis que, pendant la saison sèche, les moyennes mensuelles sont de moins de $20 \%$. La vitesse des vents est faible avec une moyenne annuelle d'environ 6 à $7 \mathrm{~km} \cdot \mathrm{h}^{-1}$. Le réseau hydrographique comprend essentiellement des koris à écoulements intermittents et des mares dont plusieurs sont temporaires et semi-permanentes, les rares mares permanentes étant celles soutenues par l'émergence des eaux souterraines.

\section{Contexte géologique}

Les principales formations géologiques rencontrées dans la zone sont des séries gréso-argileuses du Continental Terminal, les alluvions et les dépôts superficiels du Quaternaire. Sur le plan hydrogéologique, les nappes facilement accessibles par des forages peu profonds, des puits et des puisards, se rencontrent dans le Continental Terminal (CT3) et dans les alluvions, ces nappes étant parfois en continuité hydraulique.

En ce qui concerne l'aspect géomorphologique, le paysage présente une succession de plateaux latéritiques et de vallées fossiles sableuses dont les dénivelés n'excédent pas $100 \mathrm{~m}$. La végétation naturelle, constituée par une savane arborée, est actuellement déboisée, laissant actuellement la place à des sols nus entièrement colonisés par les cultures pluviales (mil et sorgho).

\section{Matériel et méthodes}

\section{Matériel}

Le matériel est composé par :

\section{Les Données}

- Les données météorologiques sont constitués des hauteurs pluviométriques de la période 1950-2015 de la station synoptique de Niamey Aéroport, des relevés de la température, des hauteurs de l'évapotranspiration et des vitesses de vents ;

- Les paramètres hydrodynamiques, les données physico-chmiques et isotopiques issus de suivis conduits par des Directions Régionales de l'Hydraulique, de mémoires de thèses de doctorat, de publications des programme Hapex Sahel et AMMA et des rapports techniques ;

- Les cartes topographiques, hydrogéologiques et thématiques ;

- Les résultats des enquêtes de terrain. 


\section{Les Outils}

- les ouvrages destinés aux prélèvement des échantillons d'eau pour des analyses physicochimiques et isotopiques dans différents laboratoires;

- les conductimètres, les sondes électriques pour des mesures des conductivités et des niveaux statiques sur le terrain ;

- les différents appareils servant aux analyses chimiques et isotopiques dans différents laboratoires ;

- les fiches d'enquêtes pour recueullir des informations sur le terrain ;

- les logiciels ArcGis 10.5, Grapher 12 et Diagramme 4.0.

\section{Méthodes}

L'approche méthodologique repose sur les techniques d'acquisition et de traitement des données :

\section{Acquisition de données}

- Les facteurs climatiques (Pluie, $\mathrm{T}^{\circ}, \mathrm{ETP} .$. ) de la station synoptique de Niamey Aéroport sont fournies par la Direction de le Météorologie Nationale $(\mathrm{DMN})$. Cette station est relativement proche et sa zone de déserte couvre pratiquement la zone d'étude.

- Suivis des paramètres hydrodynamiques : il s'agit essentiellement des relevées de niveaux statiques et dynamiques de la nappe phréatique effectués au cours de cette étude et ceux existants. Ainsi, sur le réseau piézométrique existant, il a été sélectionné une quarantaine d'ouvrages (Fig. 2) composés majoritairement de puits villageois et pastoraux mais certains sont des piézomètres réalisés dans le cadre de cette étude. Les relevés des niveaux statiques ont été effectués mensuellement à l'aide d'une sonde électrique 
durant plus de 4 ans. Pour les piézomètres, des enregistrements automatiques ont été effectués en plus des mesures ponctuelles.

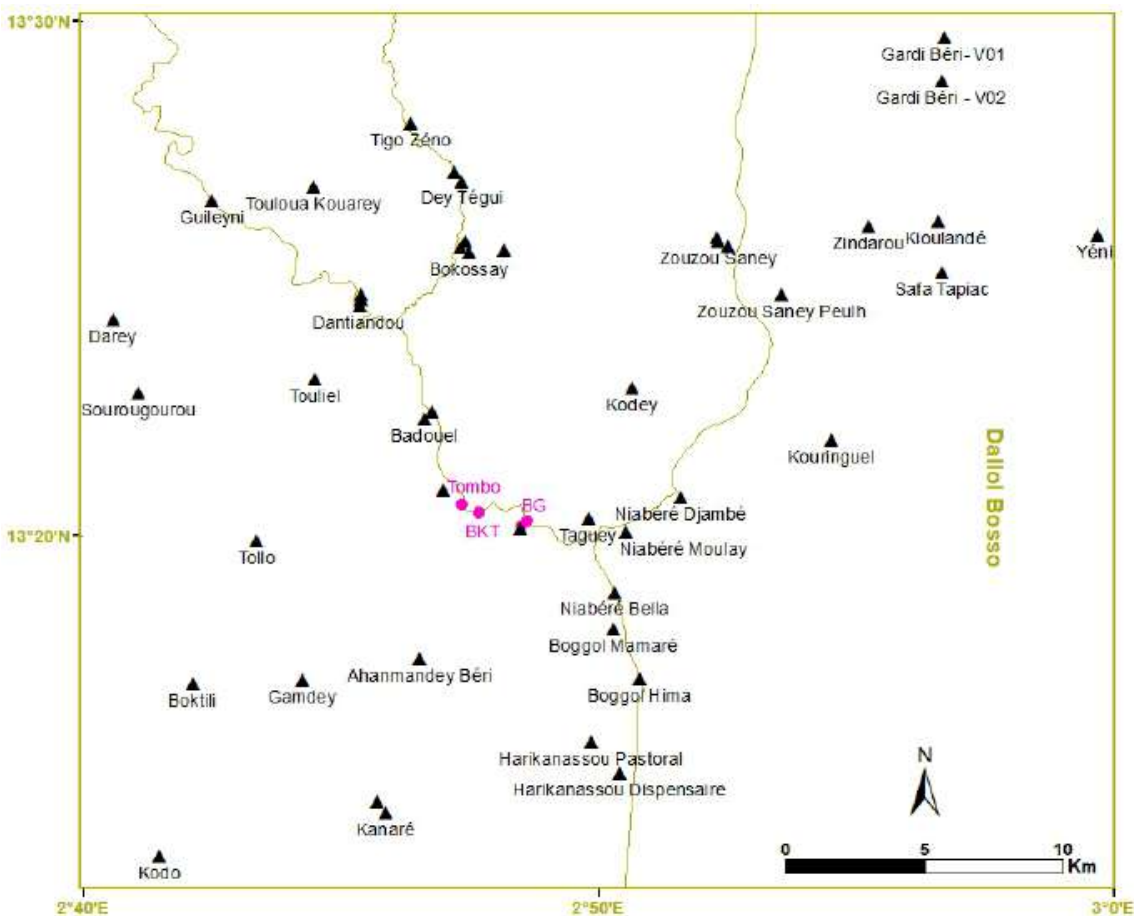

Figure 2. Réseau de mesures piézométriques mensuelles dans les ouvrages de captage de la nappe. $\boldsymbol{\Delta}$ : puits cimentés, $\bullet$ : piézomètres à proximité de mare.

- $\quad$ Suivis des paramètres physico-chimiques et isotopiques

$\checkmark$ Les paramètres physiques $\left(\mathrm{T}^{\circ}\right.$, conductivité, $\left.\mathrm{pH}\right)$ ont été relevés à chaque campagne de mesures des niveaux statiques ;

$\checkmark$ Des campagnes de prélèvement ont été effectuées pour les analyses physico-chimiques dans des puits, des piézomètres et des mares répartis judicieusement sur toute la zone d'étude. Les prélèvements d'eau ont été effectués à l'aide d'une puisette attachée à une corde ; l'eau de la puisette est versée dans un flacon préalablement rincée avec celle-ci. Les paramètres physico-chimiques (conductivité, température) étaient systématiquement mesurés. Les échantillons d'eau ont été stockés dans des flacons en Nalgène et conservés à $4^{\circ} \mathrm{C}$. Les analyses des éléments majeurs et des traces ont été réalisées au laboratoire Hydrosciences Montpellier (HSM) par ICP-MS fournissant une incertitude sur les mesures inférieure à $5 \%$.

$\checkmark$ Les prélèvements d'eau pour analyse des teneurs isotopiques ont été réalisés dans des flacons en verre ambré et concervés à $4{ }^{\circ} \mathrm{C}$. Les teneurs en isotopes stables $\left({ }^{18} \mathrm{O}\right.$ et $\left.{ }^{2} \mathrm{H}\right)$ ont été déterminées au Laboratoire Hydrosciences de Montpellier (LAMA) par spectrométrie de masse, utilisant la méthode Dual Inlet, avec équilibre de $\mathrm{CO}_{2}$ pour le ${ }^{18} \mathrm{O}$ et le flux continu 
d'hélium par réduction de l'eau sur chrome dans un four à $1050{ }^{\circ} \mathrm{C}$ pour le deutérium. La précision des résultats est de $0,05 \%$ vs. SMOW pour $\delta^{18} \mathrm{O}$ et de $0,8 \%$ vs. SMOW pour $\delta^{2} H$. Le choix de ces deux isotopes est dû au fait qu'ils sont les meilleurs traceurs (partie intégrante de la molécule d'eau) et plus faciles à analyser à moindre couts.

$\checkmark$ Des fiches d'entretien ont été élaborées en vue de collecter des informations quantitatives et qualitatives sur les usages de l'eau souterraine (irrigation, exploitation de natron, ..) ainsi que sur la dynamique des mares et de la nappe (remontée de la nappe, apparition de nouvelles mares permanentes, etc.). L'enquête a porté sur 60 agriculteurs dans 3 villages (Tombo, Boula Kouara Tégui et Boula Gandatché) à l'extrémité aval du Kori de Dantiandou en janvier 2014. Le questionnaire est composé de 56 questions à réponse prédirigées. Elles étaient aussi bien quantitatives que qualitatives. Les fiches ont été remplies par entrevues avec les chefs de ménage pratiquant l'irrigation et l'exploitation du natron.

\section{Traitement de données}

a) Les paramètres climatiques

Il a été déterminé la moyenne fixe sur 20 ans pour la période humide (1950-1969) et sur 30 ans pour la période sèche (1970-1998). Il a été calculé l'indice pluviométrique standardisé (IPS), dont la détermination est exprimée

$$
\text { IPS }=\frac{P i-P m o y}{\sigma}
$$

par l'équation suivante (Nicholson, 1983) :

où : Pi : désigne la hauteur des précipitations annuelles de l'année $\mathrm{i}$; Pmoy : désigne la valeur moyenne interannuelle de la pluviométrie sur la période

étudiée et $\sigma$ : correspond à la valeur interannuelle de l'écart type de la pluviométrie sur la période étudiée.

À partir des indices calculés indiquant les années déficitaires et excédentaires, un diagramme permettant de suivre l'évolution interannuelle de la pluviométrie est dressé.

Pour la température, l'humidité relative, l'ETP et l'insolation, les moyennes annuelles sur les périodes d'observation sont calculées suivi des différents graphiques. Concernant le vent, les valeurs moyennes mensuelles sont calculées et ensuite comparées entre elles.

\section{b) Les paramètres hydrodynamiques}

Des mesures ponctuelles et des enregistrements effectués dans les puits et les piézomètres ont permis de collecter chaque mois plusieurs données ayant permis d'élaborer des graphiques d'évolution de la nappe, et d'effectuer des comparaisons entre les relevés manuels et les enregistrements automatiques. 


\section{c) Les paramètres physico-chimiques et isotopiques}

$\checkmark$ Paramètres physiques : il a été déterminé les gammes de variations, les valeurs moyennes, les écart-types et ensuite il a été dressé des cartes thématiques de répartition spatiales des conductivités.

$\checkmark$ Les éléments chimiques : la balance ionique a été d'abord définie, qui doit être inférieure ou égale à $5 \%$ pour être considérée comme acceptable. Ensuite, une classification des éléments a été effectuée pour évaluer les proportions en pourcentage, à savoir les formules ioniques des eaux. La présentation graphique des éléments chimiques sur le diagramme de Piper a été réalisée afin de déterminer les faciès chimiques des eaux.

$\checkmark$ Les teneurs en isotopes stables $\left({ }^{18} 0\right.$ et en D) des ressources en eau (eau de pluie, de surface et souterraines) ont permis de :

- Déterminer les moyennes pondérales des teneurs en isotopes stables $\left({ }^{18} \mathrm{O}, \mathrm{D}\right)$ des précipitations actuelles au niveau régional et sous régional ;

- Élaborer un diagramme montrant la relation entre la profondeur des niveaux statiques et les teneurs des eaux souterraines en ${ }^{18} \mathrm{O}$;

- Projeter les teneurs en ${ }^{18} \mathrm{O}$ vs $\mathrm{D}$ des eaux sur la Droite Météorique Locale (DML) afin de déterminer les périodes de recharge des eaux.

\section{d) Les enquêtes de terrain}

Les données issues des fiches d'enquêtes sont traitées par analyses bivariées, en test de khi-deux, pour les données qualitatives, et par analyse de la variance (ANOVA) à un facteur, pour les données quantitatives.

\section{Résultats et discussion}

\section{Evolution des paramètres climatiques}

a) Variation annuelle des hauteurs des précipitations

Pour les précipitations recueillies à la station Niamey Aéroport qui est proche de la zone d'étude, les hauteurs pluviométriques annuelles varient de 400 et $700 \mathrm{~mm} / \mathrm{an}$, avec une valeur moyenne interannuelle de $560 \mathrm{~mm}$ et un écart type de $135 \mathrm{~mm}$, pour la période 1950-2015 (Fig. 3). Ces précipitations montrent une forte variabilité spatio-temporelle, illustrée par la valeur de l'écart-type. Ce résultat confirme ceux de certains auteurs ayant montré que le Sahel est l'une des régions du monde où la variabilité interannuelle des précipitations est la plus marquée (Nicholson and Grist, 2001).

b) Variabilité interannuelle des précipitations

Le diagramme établi à partir des indices de précipitations (Nicholson, 1983) pour la période $1950-2015$, permet de distinguer 3 phases :

- une première phase humide allant de 1950 à 1969 , avec une moyenne de $645 \mathrm{~mm}$;

- une deuxième phase sèche allant de 1970 à 1998, avec une moyenne de $500 \mathrm{~mm}$. En effet, les déficits pluviométriques annuels ont atteint $40 \%$ par rapport à la période humide 1950-1969 (Fig. 3). 
- une troisième phase ayant débuté depuis la fin des années 1990 semble être caractérisée par un retour à des conditions humides comme l'avait déjà relevé Lebel \& Ali, (2009). En effet, dans la région centrale du Sahel (SudOuest Niger), les précipitations annuelles ont augmenté environ de $10 \%$ par rapport à la période sèche 1970-1998; néanmoins les hauteurs moyennes annuelles restent inférieures à celles de la période humide 1950-1969.

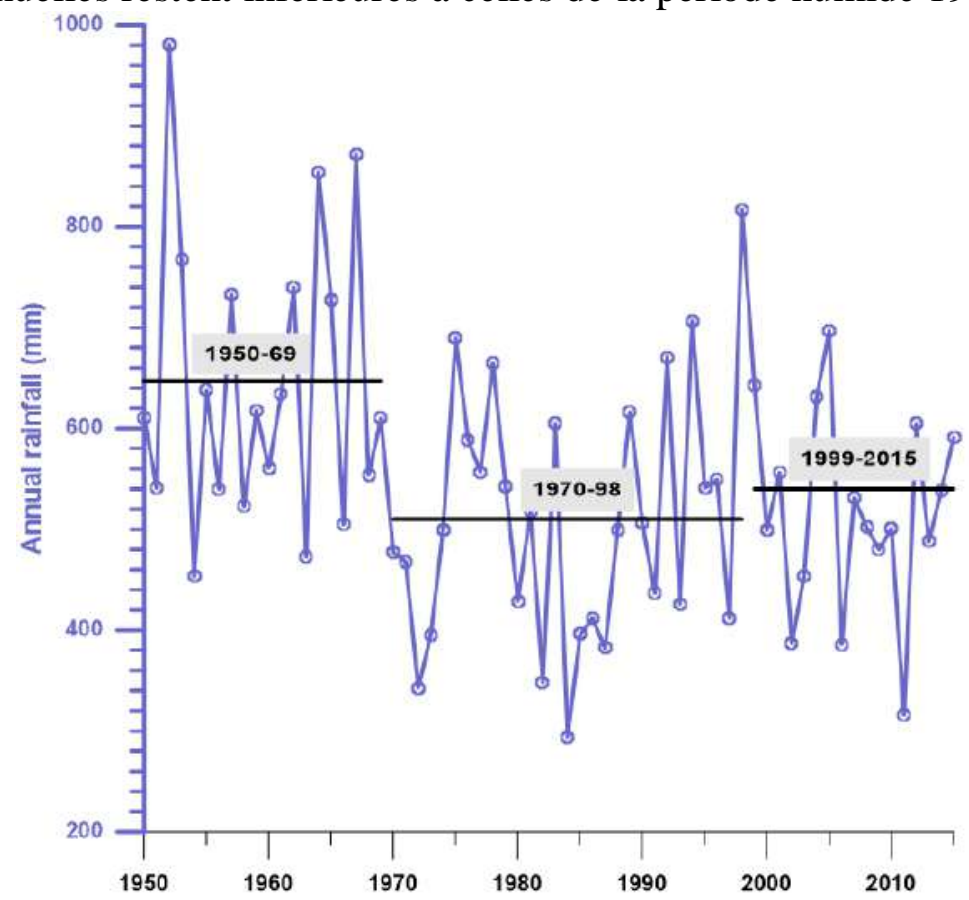

Figure 3. Moyennes interannuelles des précipitations pour la phase humide (1950-1969), sèche (1970-1998) et relativement humide (1999-2015).

c) Autres paramètres climatiques $\left(T^{\circ}\right.$, humidité relative, ETP, insolation, vents)

- La température moyenne interannuelle pour la période 1950-2015 est de $29^{\circ} \mathrm{C}$. Cependant, il a été observé une augmentation sensible de la température minimale au cours des années 1975 à 2009, qui serait en relation avec la variabilité climatique (Fig. 4);

- L'humidité relative, très faible d'octobre à mai correspondant à la saison sèche, montre une augmentation sensible pendant la saison pluvieuse ;

- La valeur moyenne annuelle de l'évapotranspiration potentielle (ETP), estimée à partir de l'équation de Penman par Massuel, (2005), est de 2500 mm pour la période 1950-2003 au niveau de la station synoptique de Niamey Aéroport. Les valeurs moyennes mensuelles de l'ETP restent toujours supérieures à celles des pluies, à l'exception des mois de juillet et août, laissant supposer que la recharge directe des aquifères à nappe libre ne pourrait avoir lieu que pendant ces mois. 


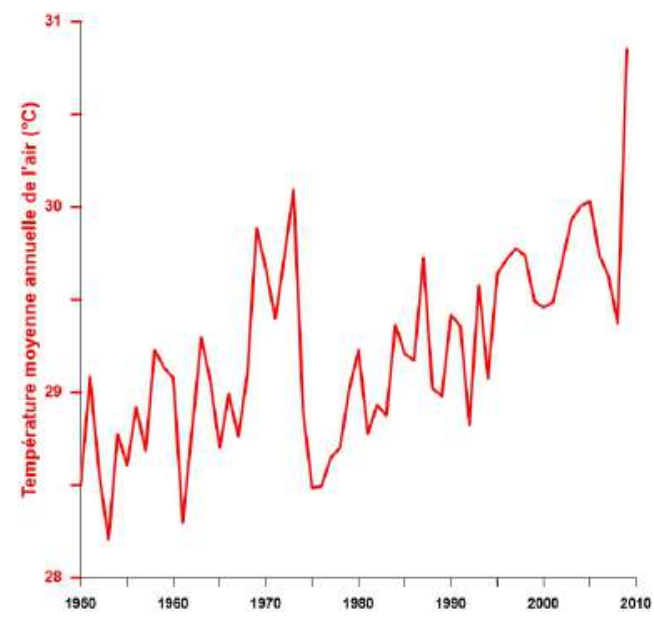

Figure 4. Températures moyennes annuelles à Niamey Aéroport (1950-2010)

\section{Évolution des niveaux statiques}

Les relevés des niveaux statiques effectuées montrent une remontée sensible de la piézométrie au niveau de plusieurs ouvrages, dont le puits de Guileyni où les fluctuations annuelles de la piézométrie peuvent varier de 20 $\mathrm{cm}$ à 2,6 m (Fig. 5). Ces fortes remontées des nappes ont entrainé, dans les zones les plus basses, l'apparition de mares permanentes alimentées par l'émergence de la surface piézométrique. La hausse de la surface piézométrique, en particulier dans les zones basses, a pour conséquence d'augmenter l'évapotranspiration qui a des effets négatifs sur les eaux souterraines, limitant même certains de leurs usages. La recharge de la nappe phréatique mise en évidence est confirmée par ailleurs par les travaux antérieurs de plusieurs auteurs dont Leduc et al., (2001) et Favreau et al., (2009), qui ont montré que celle-ci devrait avoir débuté depuis le début des années 60 .

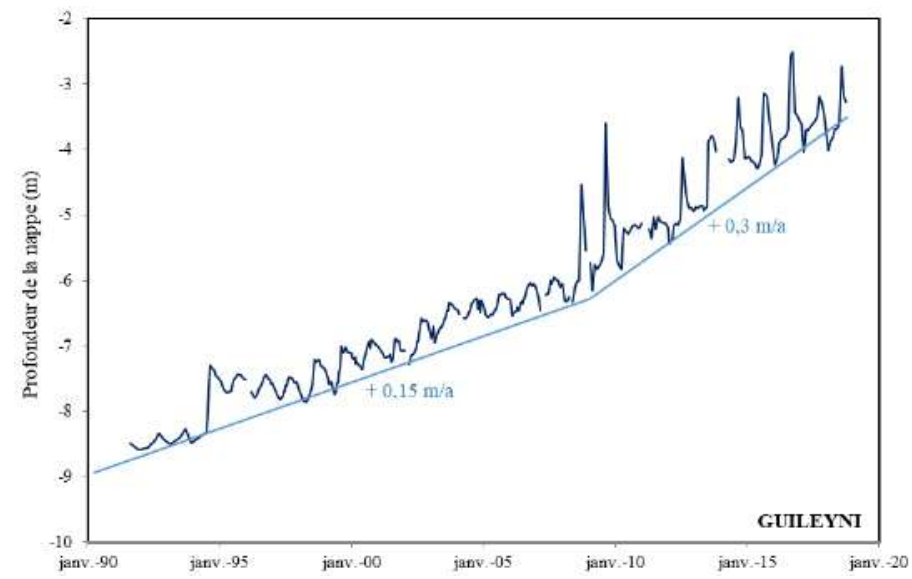

Figure 5. Evolution piézométrique à Guileyni, montrant la superposition de deux dynamiques : recharge saisonnière, hausse à long terme récemment accrue. 


\section{Evolution des caractéristiques hydrochimiques}

a) Paramètres physiques ( $T$, conductivité électrique)

Les températures des eaux de la nappe phréatique varient de $25,4^{\circ} \mathrm{C}$ à $32,1^{\circ} \mathrm{C}$. La température moyenne des eaux souterraines est de $30,6^{\circ} \mathrm{C}$. Celleci est légèrement plus élevée que celle de l'atmosphère qui est proche de 29 ${ }^{\circ} \mathrm{C}$. Il semble que la température de certaines eaux souterraines est influencée par le phénomène géothermique.

Les conductivités électriques des eaux mesurées in situ dans la nappe varient de moins de 20 à plus de $1000 \mu{\mathrm{S} . \mathrm{cm}^{-1}}^{-1}$ (Fig. 6). Les minéralisations comprises entre 20 et $150 \mu \mathrm{S} . \mathrm{cm}^{-1}$ sont rencontrées dans les zones situées en dehors du Dallol Bosso et du Kori de Dantiandou. Elles semblent caractériser la minéralisation naturelle de la nappe. Par contre, les minéralisations variant de 150 à plus de $1000 \mu \mathrm{S} . \mathrm{cm}^{-1}$ sont observées dans les vallées du Dallol Bosso et du Kori de Dantiandou, montrant ainsi que cette augmentation très importante de la minéralisation est liée à des phénomènes de pollution (ElbazPoulichet et al., 2002) et/ou d'évapotranspiration importante, d'autant plus que la nappe concernée se trouve à une très faible profondeur $(0$ à $10 \mathrm{~m})$. Ces fortes minéralisations se rencontrent également dans les eaux des mares, traduisant ainsi un phénomène de lessivage des sols déjà salinisés et d'évaporation de ces eaux. Il est à noter que ces eaux ainsi minéralisées deviennent inaptes à plusieurs usages dont la consommation humaine et, surtout, l'irrigation, empêchant aux populations de profiter des apports annuels des eaux provenant de la recharge de la nappe phréatique

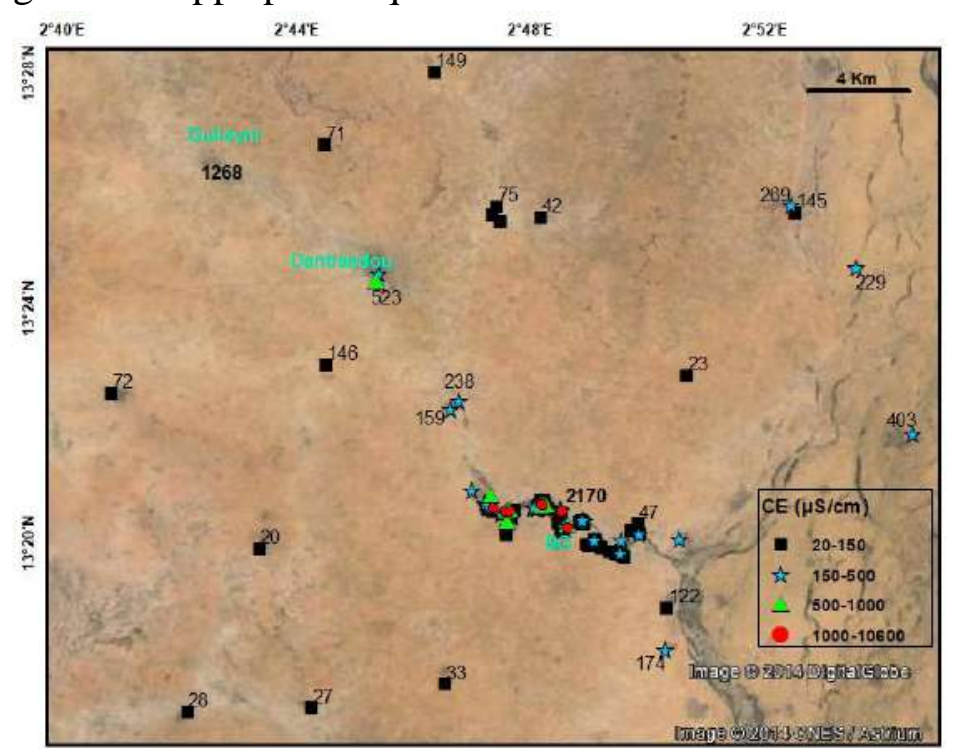

Figure 6. Répartition spatiale de la conductivité électrique dans la nappe à méso-échelle (février 2015) 


\section{b) Paramètres chimiques}

Les formules ioniques des eaux montent que :

Pour les cations : $\mathrm{Na}^{+}>\mathrm{K}^{+}>\mathrm{Ca}^{2+}>\mathrm{Mg}^{2+}$;

Pour les anions : $\mathrm{HCO}_{3}{ }^{-}>\mathrm{NO}_{3}{ }^{-}>\mathrm{Cl}^{-}>\mathrm{SO}_{4}{ }^{2-}$.

Dans les eaux souterraines comme dans les eaux de surface, le cation dominant et l'anion dominant sont respectivement $\mathrm{Na}^{+}$et $\mathrm{HCO}_{3}{ }^{-}$, ce qui semble montrer l'effet de l'évaporation. Par ailleurs, la présence des nitrates qui occupent la deuxième place parmi les anions semble indiquer que ces eaux subissent des phénomènes de pollution. La minéralisation totale des eaux varie de 115 à 450 mg dans la nappe et de 1065 à $2558 \mathrm{mg}$ pour les mares permanentes, ce qui montre que cette minéralisation est particulièrement liée au phénomène d'évaporation et confirme ce qui a été observé au niveau de la conductivité. Par conséquent, l'origine principale de la minéralisation semble refléter l'évaporation des eaux et/ou le lessivage des sols que le sens d'écoulement des eaux.

La représentation des éléments chimiques sur un diagramme de Piper (Fig. 7) montre les principaux faciès suivants :

- faciès de types bicarbonatés sodique, potassique et calcique ;

- faciès de types chlorurés ou nitratés sodique, potassique et calcique ;

- faciès de types mixtes.

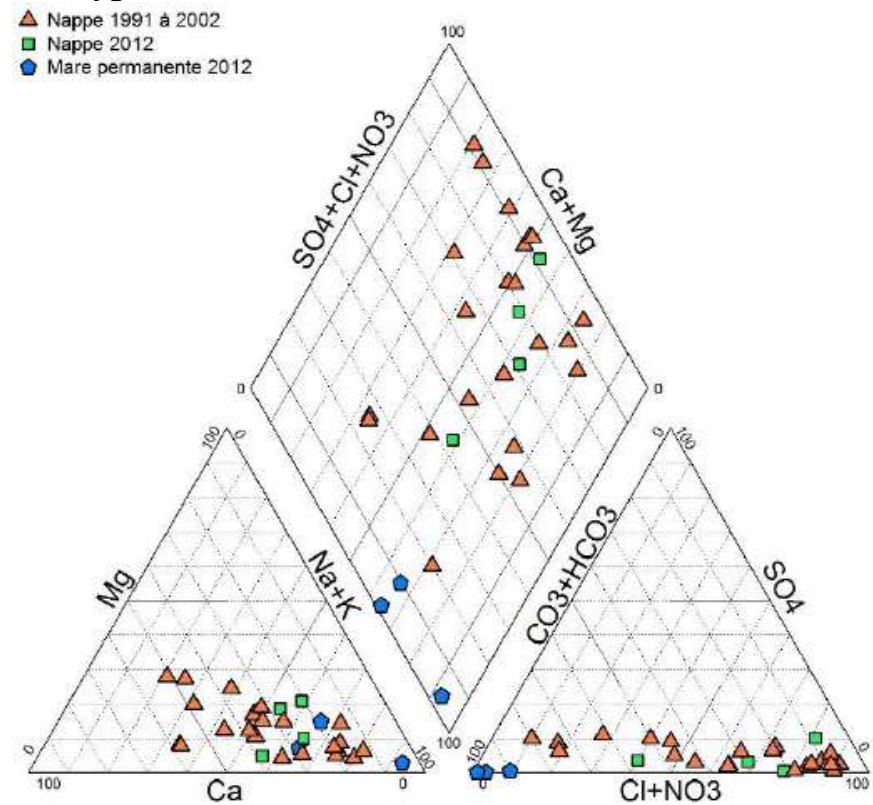

Figure 7. Distribution des ions majeurs dans la nappe phréatique et dans les mares permanentes 


\section{Variabilité des teneurs en isotopes stables des eaux}

a) Teneurs isotopiques des eaux souterraines et de surface

Les teneurs en isotopes $\left({ }^{18} \mathrm{O}, \mathrm{D}\right)$ portées sur le diagramme $\delta^{2} \mathrm{H}$ vs $\delta^{18} \mathrm{O}$ (Fig. 8) montre que certaines eaux souterraines ont une faible composante actuelle, d'autres par contre sont des mélanges des eaux actuelles et anciennes, et enfin on a un troisième groupe d'eau ayant subi un enrichissement de leurs teneurs isotopiques intervenu au cours de leur infiltration, mais probablement dans la nappe, suite à une évaporation directe. Cette dernière hypothèse semble être confirmée par les concentrations en isotopes lourds des eaux de surface atteignant parfois plus de $+20 \%$ vs SMOW en ${ }^{18} \mathrm{O}$.

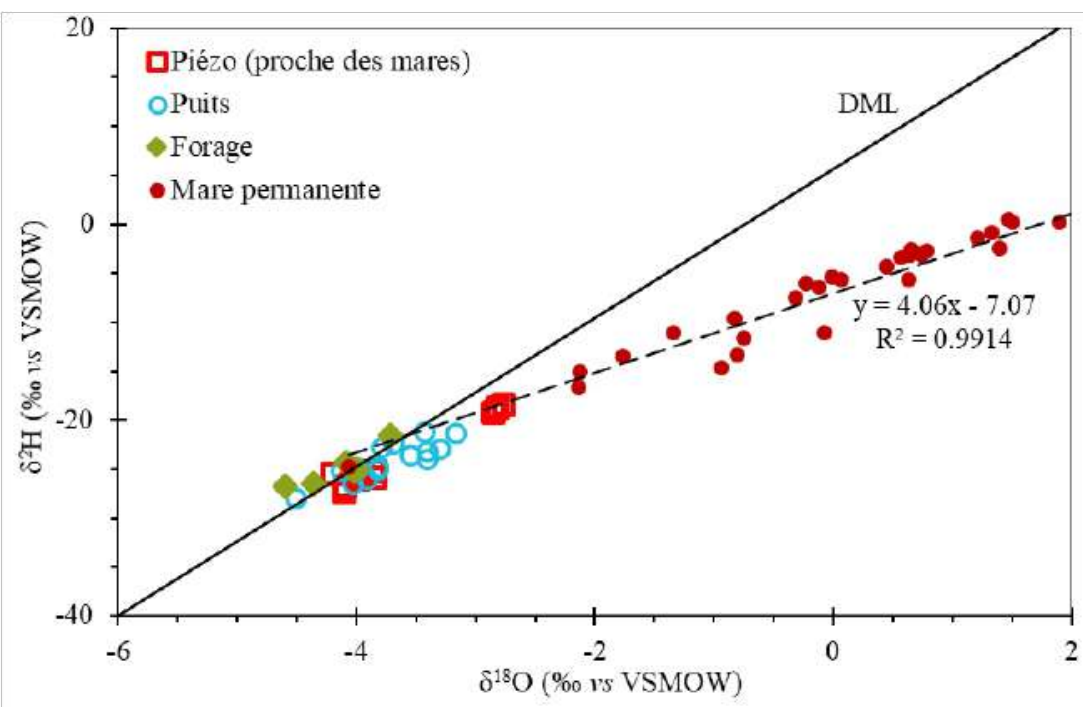

Figure 8. Diagramme $\delta^{2} H v s \delta^{18} O$ pour les eaux de surface et souterraines.

b) Relation teneurs isotopiques et profondeur des ouvrages hydrauliques

L'examen du graphique des teneurs isotopiques en fonction de la profondeur de la nappe (Fig. 9) montre que :

- Pour les profondeurs inférieures à $10 \mathrm{~m}$ : les teneurs en ${ }^{18} \mathrm{O}$ varient de -4 à $-2 \%$. Les valeurs proches de $-4 \%$ peuvent être le résultat d'une infiltration rapide des précipitations, car elles sont proches de la valeur pondérale qui est de $-4 \%$. Par contre pour ce qui concerne les autres eaux, on observe un enrichissement montrant que les eaux ont subi une évaporation, notamment pour les eaux des piézomètres qui se trouvent parfois à moins d' 1 m de la surface du sol.

- Pour les profondeurs supérieures à $10 \mathrm{~m}$ : Les teneurs isotopiques sont plus appauvries, avec des valeurs généralement comprises entre -4 et $-5 \%$ en ${ }^{18} \mathrm{O}$. Cela prouve qu'on est en face des eaux rechargées directement par les précipitations actuelles ou des mélanges d'eau provenant de celles qui sont 
récentes et anciennes. Toutefois, pour ces ouvrages dont la profondeur est supérieure à $10 \mathrm{~m}$, il a été retrouvé des eaux plus enrichies en ${ }^{18} \mathrm{O}$ (de l'ordre $-3 \%$ ), cela peut s'expliquer par une recharge indirecte à partir des eaux évaporées des mares. En effet, on observe de l'infiltration quand la mare monte très vite et déborde pendant les grandes averses.

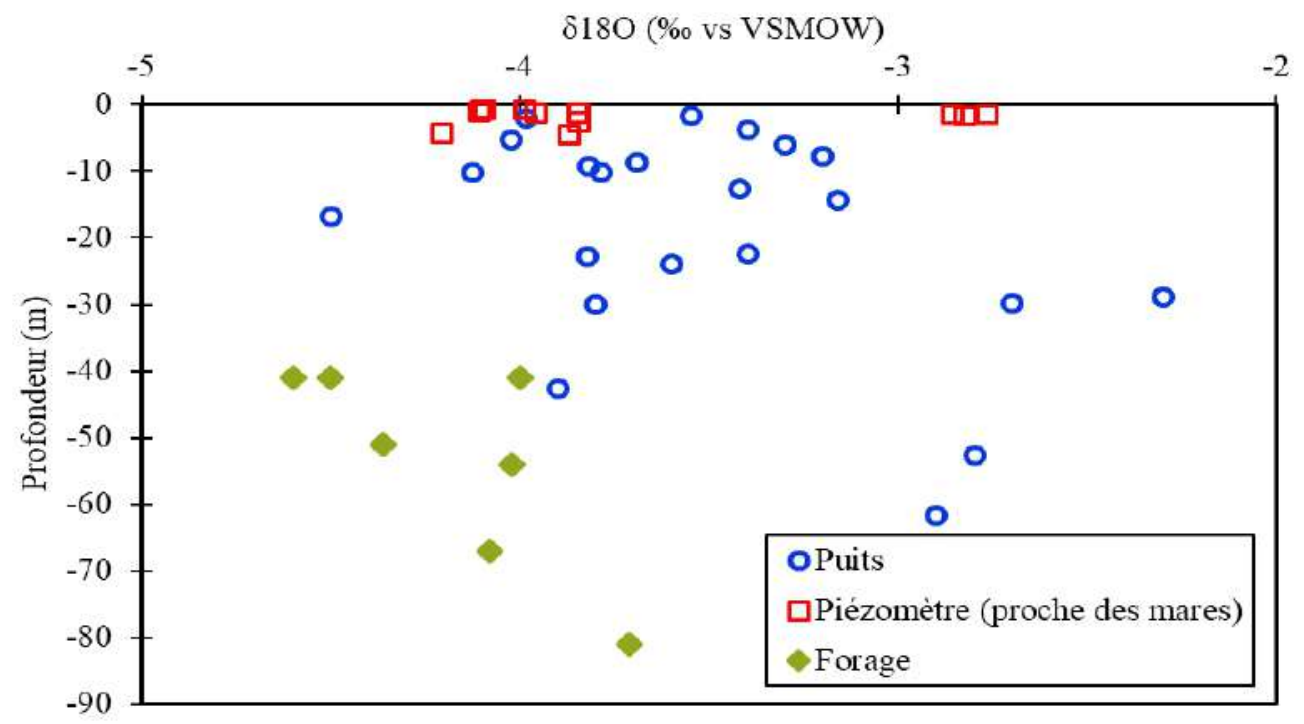

Figure 9. Evolution des teneurs isotopiques en fonction de la profondeur de la nappe

\section{Résultats des enquêtes}

Les villageois ont spontanément reconnu la remontée de la nappe sur ces dernières décennies, ainsi que l'apparition récente des mares permanentes. Cette remontée de la nappe s'est traduite par un développement accru de l'irrigation car les niveaux sont plus proches et facilitent l'exhaure. Néanmoins ils ont relevé eux-mêmes que les eaux deviennent très salées à certaines périodes et détruisent les cultures. Il a été également observé un développement de la production du natron que les populations lient à la remontée des eaux et à la salinisation des sols.

\section{Impacts de la recharge sur les ressources en eau souterraines}

L'étude de la piézométrie et des teneurs isotopiques des eaux ont montré que la nappe phréatique de Kori de Dantiandou reçoit une recharge directe et indirecte relativement importante entrainant un rehaussement des niveaux piézométriques jusqu'à la sub-surface du sol et cela malgré les conditions climatiques relativement rudes dans la bande sahélienne. Malheureusement, suite aux phénomènes d'évaporation, les eaux subissent une forte minéralisation limitant leurs usages pour satisfaire les besoins en eau des populations, notamment l'irrigation. Les zones les plus proches des mares 
connaissent également une augmentation saisonnière très forte de la salinité corrélée aux plus fortes amplitudes piézométriques. Cette augmentation de conductivité peut, exceptionnellement, aller jusqu'à un facteur 10, marquant le lessivage occasionnel des sels stockés dans la zone non-saturée par les eaux de recharge (Elbaz-Poulichet et al., 2002 ; Massuel et al., 2006). Ceci se remarque particulièrement bien avec les nitrates qui, dans un environnement sans apport notable d'engrais minéral, peuvent largement dépasser les normes de potabilité (Favreau et al., 2003).

Les eaux stagnantes permanentes présentent un risque sanitaire élevé pour les populations riveraines du fait de maladies à transmission vectorielle, en particulier le paludisme. De plus, la faible profondeur de la nappe rend les eaux souterraines particulièrement vulnérables aux contaminations issues des latrines et des zones de défécation à l'air libre.

Par ailleurs, il est observé un développement accru et récent de la petite irrigation à partir des puits cimentés ou des puisards. La faible profondeur de la nappe favorise ces nouvelles opportunités. Les phénomènes de montée des eaux et de salinisation des sols provoquent la mort des arbres (par asphyxie) et la perte des terres arables. La concentration de différents sels sur le toit de la nappe affleurante a entrainé depuis quelques années l'exploitation du natron.

En effet, plusieurs études expérimentales ont permis d'estimer l'évaporation directe à partir d'une nappe phréatique en zone semi-aride ou aride. Par exemple, dans les alluvions du fleuve Niger près de Niamey, l'évaporation est de l'ordre de $7 \mathrm{~mm}$.an ${ }^{-1}$ sous $5 \mathrm{~m}$ de couvert argilo-sableux (Taupin et al., 1991). Coudrain-Ribstein et al., (1998) ont présenté de nombreux résultats montrant les valeurs de l'évaporation d'une nappe libre en milieu semi-aride ou aride en régime permanent, en fonction de la profondeur du niveau piézométrique sous le sol; les valeurs sont de l'ordre de $26 \mathrm{~mm}$.an ${ }^{-}$ ${ }^{1}$ quand la nappe est à $2 \mathrm{~m}$ sous la surface du sol. Cette évaporation fait augmenter considérablement les sels et donc la conductivité électrique de l'eau restant dans l'aquifère.

\section{Conclusion et recommandations Conclusion}

Les observations hydrodynamiques confirment la hausse continue de la nappe phréatique, avec des niveaux piézométriques actuels particulièrement élevés. L'évaporation des eaux sub-affleurantes au sein de l'aquifère ont augmenté considérablement la conductivité électrique qui est passé de 20 à plus de $1000 \mu \mathrm{S} . \mathrm{cm}^{-1}$ au niveau des eaux souterraines et à plus de $2000 \mu \mathrm{S} . \mathrm{cm}^{-}$ ${ }^{1}$ pour les eaux de mares permanentes. Cette augmentation de la salinité est liée à l'évaporation directe à partir de la nappe phréatique qui est très sensible à proximité des mares où celle-ci est proche de la surface. Les teneurs en 
isotopes stables $\left({ }^{18} \mathrm{O},{ }^{2} \mathrm{H}\right)$ ont confirmé par ailleurs la recharge directe et indirecte de la nappe phréatique ainsi que l'évaporation que subissent les eaux souterraines et de surface. La remontée de la nappe a permis un développement de l'irrigation. Néanmoins, ces eaux ne sont pas toujours utilisables, surtout au niveau des mares permanentes récemment apparues, du fait de leur salinisation. Ces travaux ont montré également que les sols aussi se sont enrichis en différents types de sels, devenant ainsi impropres à l'agriculture, même si ces sols ont donné lieu depuis quelques années à une exploitation du natron qui représente alors une autre source de revenus pour les populations. Ainsi, la recharge de la nappe est naturelle et effective malgré les conditions climatiques difficiles dans la région, mais celle-ci présente des effets négatifs.

\section{Recommandations}

Pour permettre de préserver les ressources en eau renouvelables de la nappe phréatique du Kori de Dantiandou, il est recommandé de rabattre les niveaux statiques à l'aide de pompages intensifs pour irriguer des superficies beaucoup plus importantes, ceci grâce au soutien des autorités nationales. Une telle promotion de l'irrigation permettrait en outre d'atténuer les effets de la variabilité des précipitations sur la production de cultures pluviales.

\section{Remerciements}

Nous remercions tous ceux qui ont contribué à la réalisation de cette étude, notamment le programme AMMA-Catch ORE (2010-2015), le Laboratoire mixte international PICASS'EAU ("Projet d'impact du climat et des utilisations de l'eau en Afrique subsaharienne") et le projet européen Wassermed ("Disponibilité et sécurité de l'eau dans le sud de l'Europe et de la Méditerranée") pour leurs appuis financiers, ainsi que l'IRD au Niger pour avoir permis la réalisation des campagnes de terrain. Les villageois de Tombo, Boula Kouara Tégui et Boula Gandatché sont également remerciés pour l'accueil chaleureux de notre équipe dans les villages et les jardins.

\section{References :}

1. Coudrain-Ribstein, A., Pratx, B., Talbi, A., \& Jusserand, C. (1998). L'évaporation des nappes phréatiques sous climat aride est-elle indépendante de la nature du sol ? Comptes Rendus Académie Sci. Ser. IIA - Earth Planet. Sci. 326, 159-165.

2. Cuthbert, M.O., Gleeson, T., Moosdorf, N., Befus, K.M., Schneider, A., Hartmann, J., \& Lehner, B. (2019). Global patterns and dynamics of climate-groundwater interactions. Nat. Clim. Chang. 9, 137-141.

3. DeFries, R., \& Eshleman, K.N. (2004). Land-use change and hydrologic processes: a major focus for the future. Hydrol. Process. 18, 2183-2186. 
4. Desconnets, J.C., Taupin, J.D., Lebel, T., \& Leduc, C. (1997). Hydrology of the HAPEX-Sahel Central Super-Site: surface water drainage and aquifer recharge through the pool systems. J. Hydrol., Hapex-Sahel 188-189, 155-178.

5. Elbaz-Poulichet, F., Favreau, G., Leduc, C., \& Seidel, J.L. (2002). Major ion chemistry of groundwaters in the Continental Terminal water table of southwestern Niger (Africa). Appl. Geochem. 17, 13431349.

6. Favreau, G., Leduc, C., Seidel, J.-L., Ousmane, S. D., \& Mariotti, A. (2003). Land clearance and nitrate rich groundwater in a Sahelian aquifer, Niger. In Hydrology of the Mediterranean and Semiarid Regions, edited by E. Servat et al., IAHS Publ., 278, 163- 167.

7. Favreau, G., Cappelaere, B., Massuel, S., Leblanc, M., Boucher, M., Boulain, N., \& Leduc, C. (2009). Land clearing, climate variability, and water resources increase in semiarid southwest Niger: A review. Water Resour. Res. 45, W00A16.

8. INS. (2013). Présentation des résultats préliminaires du quatrième (4ième) recensement général de la population et de l'habitat. (RGP/H) 2012. Niamey - Niger.

9. Lebel, T., \& Ali, A. (2009). Recent trends in the Central and Western Sahel rainfall regime (1990-2007). J. Hydrol., Surface processes and water cycle in West Africa, studied from the AMMA-CATCH observing system 375, 52-64.

10. Leblanc, M.J., Favreau, G., Massuel, S., Tweed, S.O., Loireau, M., \& Cappelaere, B. (2008). Land clearance and hydrological change in the Sahel: SW Niger. Glob. Planet. Change 61, 135-150.

11. Leduc, C., Favreau, G., \& Schroeter, P. (2001). Long-term rise in a Sahelian water-table: the Continental Terminal in South-West Niger. J. Hydrol. 243, 43-54.

12. Leduc, C., Pulido-Bosch, A., \& Remini, B. (2017). Anthropization of groundwater resources in the Mediterranean region: processes and challenges. Hydrogeol. J. 25, 1529-1547.

13. Martin-Rosales, W., \& Leduc, C. (2003). Dynamiques de vidange d'une mare temporaire au Sahel: l'exemple de Banizoumbou (SudOuest du Niger). Comptes Rendus Geosci. 335, 461-468.

14. Massuel, S. (2005). Evolution récente de la ressource en eau consécutive aux changements climatiques et environnementaux du sud-ouest Niger : modélisation des eaux de surface et souterraines du bassin du kori de Dantiandou sur la période 1992-2003. Université de Montpellier 2, Montpellier.

15. Massuel, S., Favreau, G., Descloitres, M., Le Troquer, Y., Albouy, Y., \& Cappelaere, B. (2006). Deep infiltration through a sandy alluvial fan 
in semiarid Niger inferred from electrical conductivity survey, vadose zone chemistry and hydrological modelling. CATENA 67, 105-118.

16. Nicholson, S.E., \& Grist, J.P. (2001). A conceptual model for understanding rainfall variability in the West African Sahel on interannual and interdecadal timescales. Int. J. Climatol. 21, 17331757.

17. Nicholson, S.E. (1983). Sub-Saharan Rainfall in the Years 1976-80: Evidence of Continued Drought. Mon. Weather Rev. 111, 1646-1654.

18. Scanlon, B.R., Jolly, I., Sophocleous, M., \& Zhang, L. (2007). Global impacts of conversions from natural to agricultural ecosystems on water resources: Quantity versus quality. Water Resour. Res. 43, W03437.

19. Taupin, J.D., Dever, L., Fontes, J.C., Guero, Y., Ousmane, B., \& Vacher, P. (1991). Evaluation de l'évaporation à travers les sols par modélisation des profils isotopiques sous climat sahélien : exemple de la vallée du Niger. Publ. AISH 159-171.

20. Vörösmarty, C.J., Green, P., Salisbury, J., \& Lammers, R.B. (2000). Global Water Resources: Vulnerability from Climate Change and Population Growth. Science 289, 284-288. 